\title{
Numerical Investigation of Chemically Reacting MHD Flow Due to a Rotating Cone with Thermophoresis and Brownian Motion
}

\author{
C. Sulochana ${ }^{1}$, G. P. Ashwinkumar ${ }^{2}$ and N. Sandeep ${ }^{3}$ \\ Department of Mathematics, Gulbarga University, Gulbarga-585106, India \\ math.sulochana@gmail.com
}

\begin{abstract}
In this study we analyzed the momentum, heat and mass transfer behaviour of magnetohydrodynamic flow towards a vertical rotating cone in porous medium with thermophoresis and Brownian motion effects. Further, the numerical solutions are elucidated by using Runge-Kutta based shooting technique. Obtained results are validated with open literature and found an excellent agreement. The influence of nondimensional governing parameters on velocity, temperature and concentration profiles along with the skin friction factor, local Nusselt and Sherwood numbers are determined and discussed with the help of graphs and tables. Results proved that the thermophoresis and Brownian motion parameters have tendency to control the heat and mass transfer rates. It is also found that a raise in the porosity parameter develops the heat and mass transfer rate.
\end{abstract}

Keywords: MHD, Rotating cone, Thermophoresis, Brownian motion, Chemical reaction

\section{Introduction}

The study of convective heat and mass transfer due to a vertical cone is a topic of admirable interest among the researchers due to its enormous practical applications in the field of engineering and technology. Mainly in the design of spacecrafts, steam generators, nuclear reactors, power transformers, solar power collectors, etc. In view of above applications Cheng and Minkowycz [1] studied the problem of natural convection through a vertical plate. Later the problem of Cheng and Minkowycz for the case of heat and mass transfer was extended by Khair and Bejan [2]. The effect of variable Buoyancy parameter on steady mixed convection flow past a vertical cone was discussed by Hering and Grosh [3]. Himasekhar et al., [4] analyzed the double-diffusive convection flow over a vertical rotating cone with variable Prandtl numbers. Nadeem and Saleem [5] analytically discussed the unsteady double diffusive convection flow through a revolving vertical cone in a revolving fluid with magneticfield effect. Ravindran et al., [6] presented a non-similarity solution for the steady flow; in continuation of this Roy et al., [7] discussed the non-similarity solution for the unsteady flow. The researchers [8-9] studied the influence of chemical reaction on the MHD flows through different channels. The effects of thermal and mass dispersion on an unsteady mixed convective fluid motion past a rotating cone embedded in rotating fluid was presented by Roy and Anilkumar [10]. Gorla et al., [11] discussed the heat and mass transfer rates for free convective flow of a saturated nanofluid through a vertical cone immersed in a porous regime.

Thermal radiation impact on MHD nanofluid flow through a rotating cone with Brownian motion and thermophoresis parameters was theoretically analyzed by Nadeem and Saleem [12]. Ahmet Kaya [13] numerically analyzed the effects of high porosity on mixed convective heat transfer flow through a vertical cone placed in a porous media. The 
steady MHD mixed convective flow of a chemically reacting fluid through a rotating vertical cone placed in a porous regime in presence of Soret and Dufour effects was discussed by Sharma and Debozani [14]. Kabeir et al., [15] studied the combined effects of magnetic field and thermal radiation on free convection flow about a vertical cone immersed in porous medium. Moreover, the coupled heat and mass transmission by mixed convection in a fluid over a vertical surface placed in a porous media has been the topic of several studies in recent year. This interest in the topic is due to the numerous engineering applications in building thermal insulation, geothermal reservoirs, crude oil extraction, petroleum industries, underground disposal of nuclear waste, fiber insulation and filtration, etc. And in variety of engineering fields such as heat exchange devices, petroleum reservoirs, chemical catalytic reactors and processes. In view of these enormous applications Elbashbeshy et al., [16] discussed the mixed convective heat transfer flow over a vertical moving surface in a porous medium with the combined effects of magnetic field and thermal radiation. The double diffusive convection heat transfer flow through a vertical surface embedded in a porous medium with melting effects was analyzed by Cheng and Lin [17]. Makinde and Ogulu [18] analyzed the thermal radiation impact on MHD mixed convective heat and mass transfer movement through a vertical surface embedded in a porous medium. The effect of the second law of thermodynamics on double diffusive convection flow of a nanofluid through a rotating porous disk in the presence of the magnetic field was studied by Rashidi et al., [19]. Rout et al., [20] discussed the influence of chemical reaction on MHD double diffusive convection flow on a moving vertical surface with convective boundary conditions. The effect radiation and cross diffusion on the flow over a stretching surface was discussed by Raju et al., [21]. Parida et al., [22] numerically analyzed the influence of chemical reaction parameter on MHD mixed convective heat and mass transfer movement through a vertical rotating surface with heat absorption. Few studies regarding the flow, heat and mass transfer through a vertical surface are mentioned in Refs. [23-28].

In recent years the problems of chemical reaction effects on MHD flow, heat and mass transfer have attracted the attention of many researchers because of its vast applications in many chemical engineering processes; there occur a chemical reaction between foreign mass and fluid, these processes occur in numerous industrial applications such as in polymer production, food processing and manufacturing of ceramics or glassware, etc. In view of these applications Ibrahim et al., [29] discussed the combined effects of chemical reaction and radiation absorption on MHD free convective heat transfer flow through a semi-infinite moving vertical surface with heat absorption and generation. Mahdy [30] focused on the influence of chemical reaction and combined effects on the flow past a vertical cone immersed in a porous medium. Das et al., [31] examined the influence of chemical reaction and internal heat generation on the flow through an infinite moving vertical plate. Mallikarjuna et al., [32] discussed the combined effects of magnetic field and chemical reaction on mixed convection heat and mass transfer flow of a Newtonian fluid through a vertical rotating cone immersed in porous media.

To the authors knowledge no studies has been reported yet to analyse the flow, heat and mass transfer behaviour of magnetohydrodynamic flow towards a vertical rotating cone through porous medium in the presence of thermophoretic and Brownian motion effects. In this study we presented the numerical solutions by using Runge-Kutta based shooting technique. The influence of non-dimensional governing parameters on velocity, temperature and concentration profiles along with the friction factor, local Nusselt and Sherwood numbers are determined and discussed with the help of graphs and tables.

\section{Mathematical Analysis}

Consider a steady two-dimensional incompressible electrically-conducting boundary layer flow induced by a rotating vertical cone with an angular velocity $\Omega$ embedded in a 
variable porosity medium as shown in Figure 1. A uniform magnetic field is applied in $z$ direction normal to the cone surface. It is assumed that the fluid properties are to be isotropic and constant except the density variation in the buoyancy force term of the momentum equations. In this study the induced magneticfield is neglected. In addition, it is also assumed that the first order homogeneous chemical reaction along with thermophoresis and Brownian motion effects are taken into account.

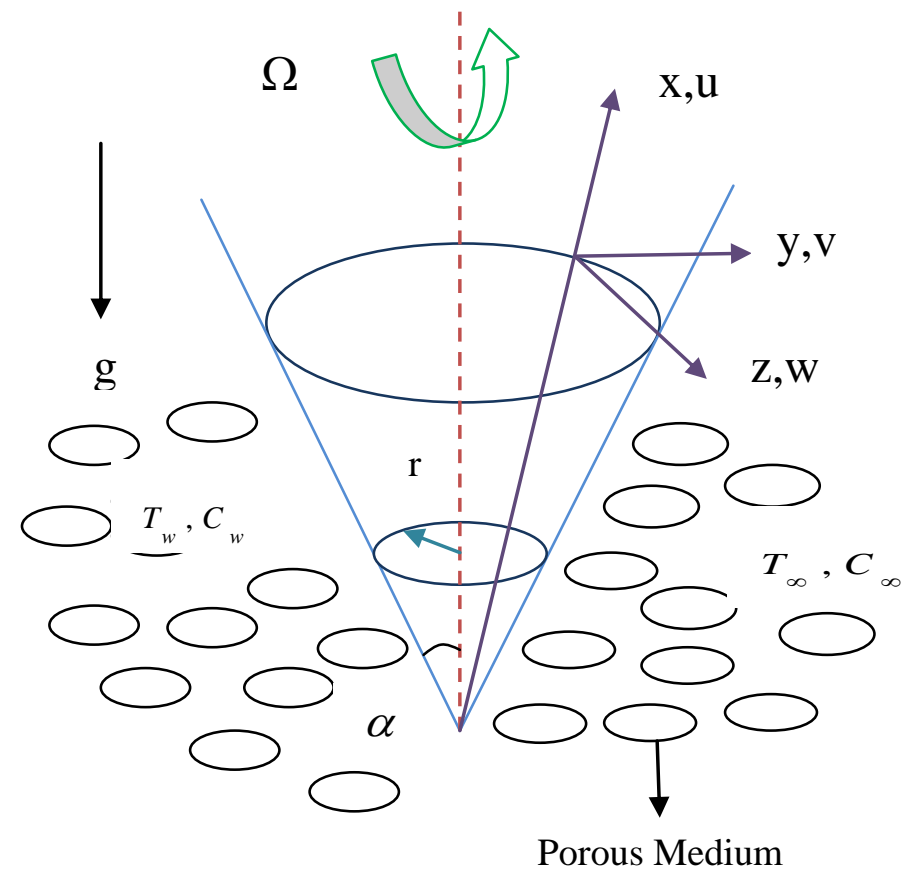

Figure 1. Physical Configuration

Under the above conditions the governing boundary layer equations are as follows (Mallikarjuna et al., [32]):

$$
\begin{aligned}
& \frac{\partial u}{\partial x}+\frac{\partial w}{\partial z}+\frac{u}{x}=0 \\
& \delta^{-2} \rho\left(u \frac{\partial u}{\partial x}+w \frac{\partial u}{\partial z}-\frac{v^{2}}{x}\right)=\delta^{-1} \mu \frac{\partial^{2} u}{\partial z^{2}}-\sigma B_{0}^{2} u-\frac{\mu}{K} u+\rho g\left(\beta_{T}\left(T-T_{\infty}\right)+\beta_{C}\left(C-C_{\infty}\right)\right) \cos \alpha, \\
& \delta^{-2} \rho\left(u \frac{\partial v}{\partial x}+w \frac{\partial v}{\partial z}+\frac{u v}{x}\right)=\delta^{-1} \mu \frac{\partial^{2} v}{\partial z^{2}}-\sigma B_{0}^{2} v-\frac{\mu}{K} v, \\
& \left.u \frac{\partial T}{\partial x}+w \frac{\partial T}{\partial z}=\frac{k_{e}}{\rho C_{p}} \frac{\partial^{2} T}{\partial z^{2}}+\tau \mid D_{B} \frac{\partial C}{\partial z} \frac{\partial T}{\partial z}+\frac{D_{T}}{T_{\infty}}\left(\frac{\partial T}{\partial z}\right)^{2}\right], \\
& u \frac{\partial C}{\partial x}+w \frac{\partial C}{\partial z}=D_{B} \frac{\partial^{2} C}{\partial z^{2}}-K_{r}\left(C-C_{\infty}\right)+\frac{D_{T}}{T_{\infty}}\left(\frac{\partial^{2} T}{\partial z^{2}}\right),
\end{aligned}
$$


The corresponding boundary conditions are

$$
\left.\begin{array}{rlrl}
u & =0, v=r \Omega, w=0, T=T_{w}(x), C=C_{w}(x) & \text { at } & z=0, \\
u & =0, v=0, T=T_{\infty}, C=C_{\infty} & \text { as } & z \rightarrow \infty
\end{array}\right\}
$$

Where $x$-axis is along a meridional section, $y$-axis is along a circular section and the $z$-axis is normal to the cone surface, $u, v$ and $w$ are the velocity components along the tangential ( $x$ ), circumferential or azimuthal ( $y$ ) and normal $(z)$ directions respectively, $\Omega$ is the angular velocity of the rotation, $r$ is radius of the cone, $\delta$ is the porosity parameter, $\rho$ is the fluid density, $\mu$ is the dynamic viscosity, $c_{p}$ specific heat at constant pressure, $g$ is the acceleration due to gravity, $\beta_{T}$ and $\beta_{C}$ are the thermal and concentration coefficients, $\alpha$ is the cone apex half angle, $K$ is the permeability of the porous medium, $k_{e}$ is the effective thermal conductivity, $K_{r}$ is the chemical reaction parameter and $D_{B}$ is the molecular diffusivity.

In order to obtain non-dimensional equations, we introduce the following nondimensional transformations,

$$
\begin{aligned}
& \eta=\left(\frac{\Omega \sin \alpha}{v}\right)^{1 / 2} z, u=x \Omega \sin \alpha f(\eta), v=x \Omega \sin \alpha g(\eta), \quad r=x \sin \alpha, w=(v \Omega \sin \alpha)^{1 / 2} h(\eta), \\
& \theta(\eta)=\frac{T-T_{\infty}}{T_{w}-T_{\infty}}, \phi(\eta)=\frac{C-C_{\infty}}{C_{w}-C_{\infty}}, T_{w}(x)-T_{\infty}=\frac{\left(T_{L}-T_{\infty}\right) x}{L}, C_{w}(x)-C_{\infty}=\frac{\left(C_{L}-C_{\infty}\right) x}{L}, \\
& D a^{-1}=\frac{v}{K \Omega \sin \alpha}, H a^{2}=\frac{\sigma B_{0}^{2} L^{2}}{\mu}, \quad \mathrm{Re}_{L}=\frac{\Omega L^{2} \sin \alpha}{v}, M=\frac{H a^{2}}{\mathrm{Re}_{L}}, \quad \operatorname{Pr}=\frac{K_{e}}{\mu C_{p}}, \\
& G r_{L}=\frac{g \beta_{T} \cos \alpha\left(T_{w}-T_{\infty}\right) L^{3}}{v^{2}}, \quad N=\frac{\beta_{c}\left(C_{w}-C_{\infty}\right)}{\beta_{T}\left(T_{w}-T_{\infty}\right)}, \lambda=\frac{G r_{L}}{\mathrm{Re}_{L}^{2}}, \quad S c=\frac{v}{D}, \quad \gamma=\frac{K_{r}}{\Omega \sin \alpha}, \\
& N b=\frac{\tau D_{B}\left(C_{w}-C_{\infty}\right)}{v}, \quad N t=\frac{\tau D_{T}\left(T_{w}-T_{\infty}\right)}{v T_{\infty}}
\end{aligned}
$$

Where $L$ being the cone slant height and $T_{L}$ being the cone surface temperature and $C_{L}$ being the cone surface concentration at the base $(x=L)$

By substituting Eq. (7) in the Eq.(1) - (5), we get

$$
\begin{aligned}
& f=-\frac{1}{2} h^{\prime}, \\
& -\delta^{-1} h^{\prime \prime}+\delta^{-2} h h^{\prime \prime}+\left(M+D a^{-1}\right) h^{\prime}+\delta^{-2}\left(-\frac{1}{2} h^{\prime^{2}}+2 g^{2}\right)+2 \lambda(\theta+N \phi)=0, \\
& \delta^{-1} g^{\prime \prime}-\delta^{-2}\left(h g^{\prime}-h^{\prime} g\right)-\left(M+D a^{-1}\right) g=0, \\
& \frac{1}{\operatorname{Pr}} \theta^{\prime \prime}+N b \theta^{\prime} \phi^{\prime}+N t \theta^{\prime 2}-\left(h \theta^{\prime}-\frac{1}{2} h^{\prime} \theta\right)=0, \\
& \phi^{\prime \prime}+\frac{N t}{N b} \theta^{\prime \prime}-S c\left(h \phi^{\prime}-\frac{1}{2} h^{\prime} \phi\right)-S c \gamma \phi=0,
\end{aligned}
$$


The corresponding boundary conditions are

$f=h^{\prime}=0, g=0, \theta=1, \phi=1 \quad$ at $\eta=0$,

$h^{\prime}=0, g=0, \theta=0, \phi=0$ as $\eta \rightarrow \infty$,

Where, prime denotes ordinary differentiation with respect to $\eta$. Here $\eta$ is the transformed coordinate $f, g$ and $h$ are the dimensionless velocity components along the tangential, azimuthal and normal directions respectively; $\theta$ is the dimensionless temperature, $\phi$ is the dimensionless concentration, $D a^{-1}$ is the inverse of Darcy number, $G r_{L}$ is the Grashof number, $\mathrm{Re}_{L}$ is the Reynolds number, $N b$ is the Brownian motion parameter, $N t$ is the Thermophoresis parameter, $\lambda$ is the dimensionless buoyancy parameter, $N$ is the buoyancy ratio such that $N<0$ corresponds to opposing flow while $N>0$ corresponds to aiding flow; $M$ is the dimensionless magnetic parameter, $H a^{2}$ is the Hartmann number, $\gamma$ is the dimensionless chemical reaction parameter, $S c$ is the Schmidt number.

The main objective of the study is to find the parameters of physical interest in the fluid flow, heat and mass transport problems are local surface skin fraction coefficient, local Nusselt and Sherwood numbers are respectively in $\mathrm{x}$ and $\mathrm{y}$ directions. These parameters are given in non-dimensional form as follows:

$$
\begin{aligned}
& C_{f x} \mathrm{Re}^{1 / 2}=-h^{\prime \prime}(0), \\
& 2^{-1} \mathrm{Re}^{1 / 2} C_{f y}=-g^{\prime}(0), \\
& \mathrm{Re}^{-1 / 2} N u_{x}=-\theta^{\prime}(0), \\
& \mathrm{Re}^{-1 / 2} S h_{x}=-\phi^{\prime}(0),
\end{aligned}
$$

\section{Results and Discussion}

In order to get the effective results, comprehensive numerical computations are carried out for various values of parameters such as Magnetic field parameter $M$, Porosity parameter $\delta$, Thermophoresis parameter $N t$, Brownian motion parameter $N b$, Chemical reaction parameter $\gamma$, Buoyancy parameter $\lambda$ that describe the flow characteristics. The system of non linear ordinary differential equations (8) to (12) with boundary conditions (13) are solved numerically by using Runge-Kutta based shooting technique. The distributions of the tangential velocity $f(\eta)$, circumferential velocity $g(\eta)$, normal velocity $h(\eta)$, temperature $\theta(\eta)$ and concentration $\phi(\eta)$ from equations (8) to (12) are clearly illustrated from figures 2 to 22 . For numerical results we considered the nondimensional values as $M=N=1, \delta=4, \lambda=10, N t=N b=0.1, S c=0.6, D a^{-1}=\gamma=0.5$. These values are kept as common in entire study except the variation in the respective figures and tables.

Figures 2-5 depicts the variations in tangential velocity profiles $f(\eta)$, circumferential velocity profiles $g(\eta)$ normal velocity profiles $h(\eta)$, temperature profiles $\theta(\eta)$ and concentration profiles $\phi(\eta)$ for different values of magnetic field parameter $M$. It is evident from Figures 2-4 that an increase in magnetic field parameter depresses the tangential and circumferential velocity; conversely there is a rise in normal velocity as increase in magnetic field parameter $M$. This is due the reason that effect of transverse magnetic field on an electrically conducting fluid creates a retarding force, known as Lorentz force. This force slows down the motion of the fluid; hence it declines the velocity of the fluid. In addition, the boundary layer thickness decreases. It is apparent 
from Figure 5 that an increase in magnetic field parameter enhances the temperature as well as concentration profiles. This may happen due to the resistive Lorentz force which opposes the fluid motion because of this heat is produced as a result there is a hike in thermal and concentration boundary layer thickness.

Figures 6-9 illustrate the effect of porosity parameter $\delta$ on velocity, temperature and concentration profiles. From Figures 6-8 it is noticed that an increase in porosity parameter $\delta$, reduces the circumferential velocity and normal velocity, while the tangential velocity reaches the maximum level for $\eta=0.5$ and reaches minimum level for increase in the value of $\eta$. The reason behind this is an increase in porosity parameter means widen the holes of the porous medium as a result the resistive forces acts opposite to the flow and there is a fall in velocity profiles. From Figure 9 it is clearly seen that an increase in porosity parameter $\delta$ reduces the both thermal and concentration boundary layer thicknesses.

Figures 10-12 reveal the effect of thermophoresis parameter $N t$ on tangential velocity profiles $f(\eta)$, normal velocity profiles $h(\eta)$, temperature and concentration profiles. From Figures 10 and 11 it is clearly observed that an increase in the thermophoresis parameter $N t$ improves the tangential velocity where as the normal velocity reduces with rise in thermophoresis parameter $N t$. This is evident from the Figure 12 that an increase in thermophoresis parameter $N t$ showed the mixed performance in temperature and concentration profiles. We have seen an opposite results to the above with an increase in the Brownian motion parameter, which are shown in Figures 13-15. This is due to the fact that Brownian motion parameter acts to warm the fluid in the boundary layer.

Figures 16-18 present the variations in tangential velocity profiles $f(\eta)$, normal velocity profiles $h(\eta)$, temperature profiles $\theta(\eta)$ and concentration profiles $\phi(\eta)$ for different values of chemical reaction parameter $\gamma$. It is evident that an increase in the chemical reaction parameter $\gamma$ retards the tangential velocity $f(\eta)$ this reduction happens because of the fluid behavior near to the cone surface when the chemical reaction parameter increases. From Figure 17 it is clear that an increase in the chemical reaction parameter $\gamma$ leads to enhancement in normal velocity profiles $h(\eta)$, this is because of the accelerating fluid motion in normal direction by increasing chemical reaction parameter. It is apparent from Figure 18 that an increase in chemical reaction parameter improves temperature profiles; conversely it declines the concentration profiles. Therefore we conclude that development in chemical reaction parameter leads to enhancement in thermal boundary layer in $y$ and $z$ direction while it depreciates concentration boundary layer thickness.

Figures 19-22 depict the effect of buoyancy parameter $\lambda$ on velocity, temperature and concentration profiles. It is clear that an increase in buoyancy parameter enhances the tangential velocity profiles, conversely depreciates the circumferential velocity and normal velocity. Buoyancy parameter is the ratio of the buoyancy to the viscous forces; this will accelerates the fluid and works as a pressure gradient. It is evident from Figure 22 that an increase in buoyancy parameter depresses the temperature profiles and concentration profiles. This may happens due to the presence of buoyancy forces, the viscous forces will be negligible.

Table 1 shows the comparison of the present results with the existed results. We found an excellent agreement of the present results with the existed literature. This proves the validity of the present results along with the accuracy of the numerical technique we used in this study.

Table 2 depicts the effect of dimensionless governing parameters on friction factors, local Nusselt and Sherwood numbers. It is evident that magneticfield parameter have tendency to reduce the heat and mass transfer rate along with friction factor in normal 
direction. But we have seen an opposite results to above with an increase in the porosity parameter. A raise in the thermophoresis and Brownian motion parameters respectively enhances the heat and mass transfer rates. Buoyancy parameter have tendency to enhance the friction factors along with heat and mass transfer rate.

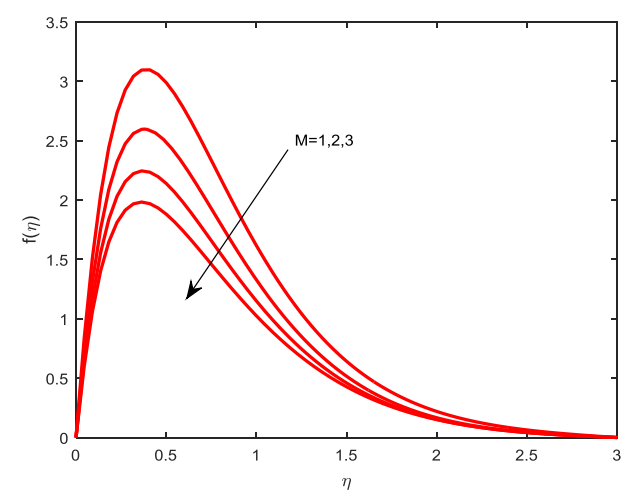

Figure 2. Velocity Profiles $f(\eta)$ for Various Values of Magnetic Field Parameter ${ }_{M}$

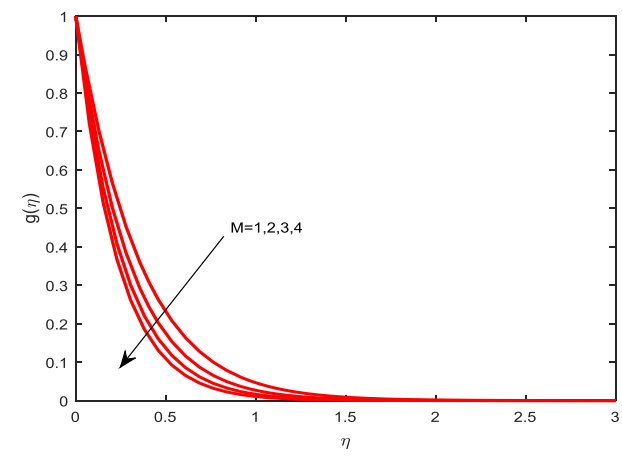

Figure 3. Velocity Profiles $g(\eta)$ for Various Values of Magnetic Field Parameter $M$

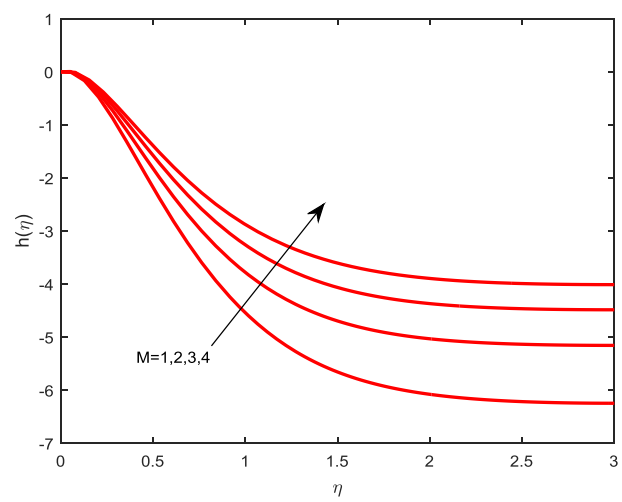

Figure 4. Velocity Profiles $h(\eta)$ for Different Values of Magnetic Field Parameter $M$

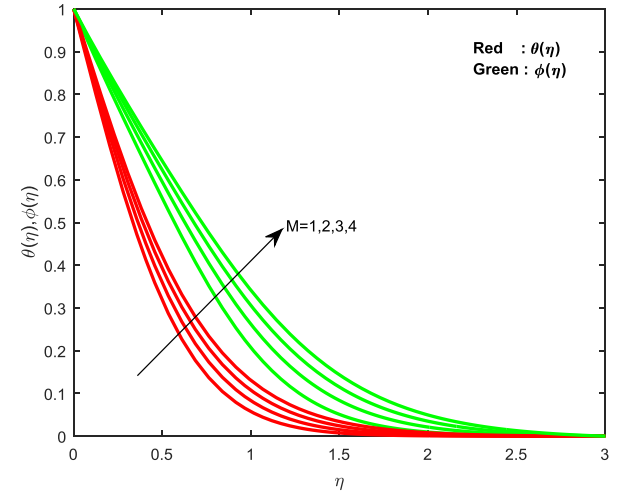

Figure 5. Temperature Profiles $\theta(\eta)$ and Concentration Profiles for Different Values of Magnetic Field Parameter ${ }_{M}$

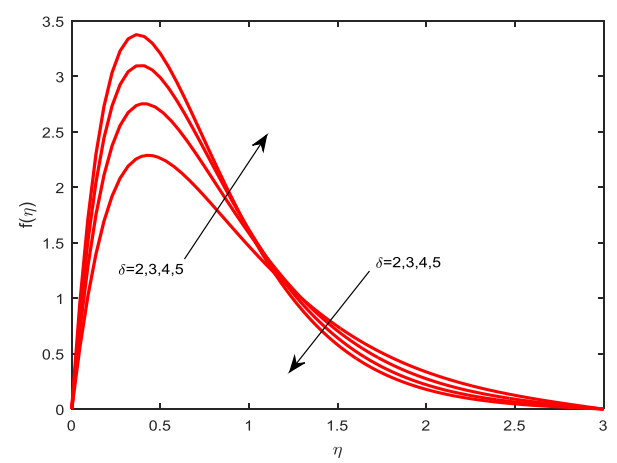

Figure 6. Velocity Profiles $f(\eta)$ for Various Values of Porosity Parameter $\delta$

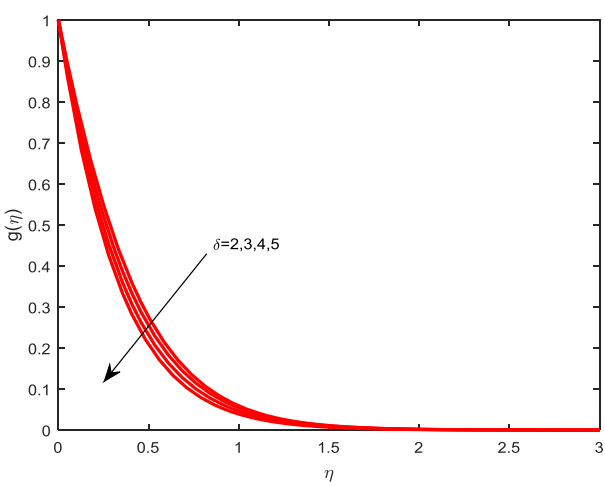

Figure 7. Velocity Profiles $g(\eta)$ for Various Values of Porosity Parameter $\delta$ 


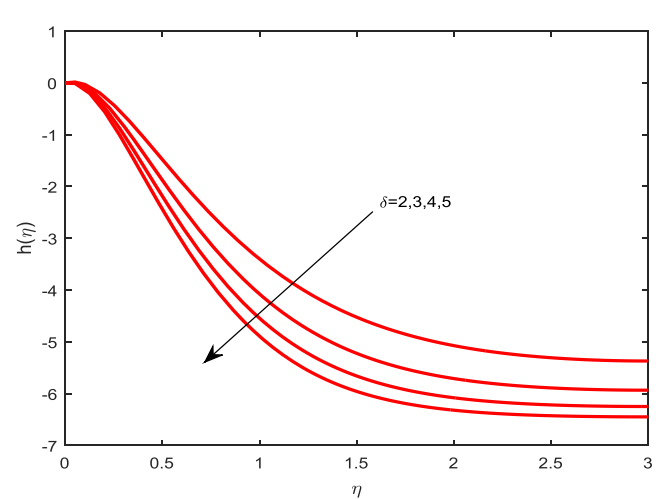

Figure 8. Velocity Profiles $h(\eta)$ for Various Values of Porosity Parameter $\delta$

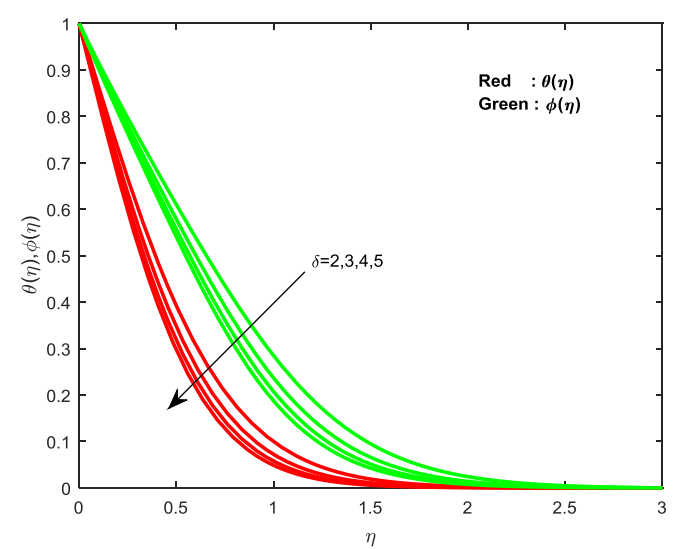

Figure 9. Temperature Profiles $\theta(\eta)$ and Concentration Profiles $\phi(\eta)$ for Different Values of Porosity Parameter $\delta$

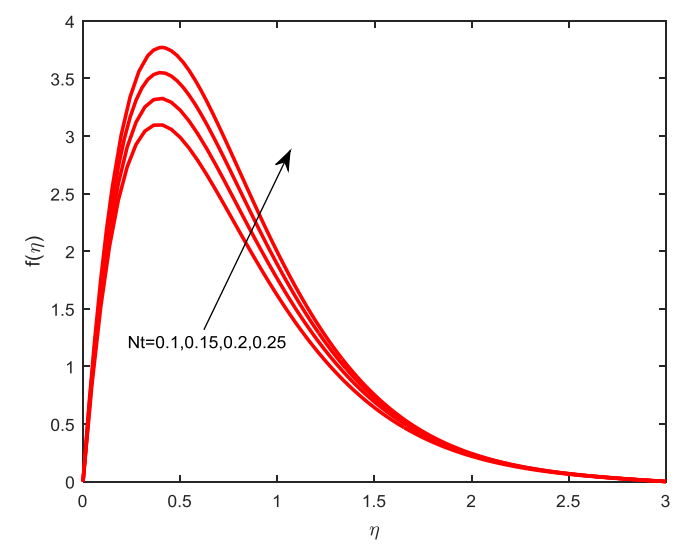

Figure 10. Velocity Profiles $f(\eta)$ for Various Values of Thermophoresis Parameter $\mathrm{N} t$

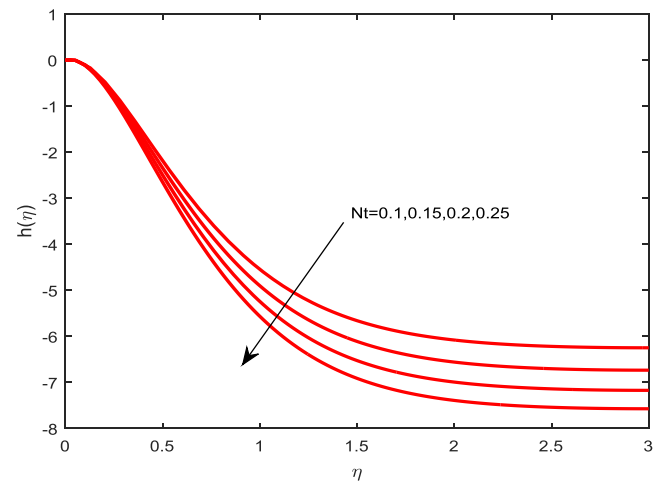

Figure 11. Velocity Profiles $h(\eta)$ for Various Values of Thermophoresis Parameter $\mathrm{N} t$

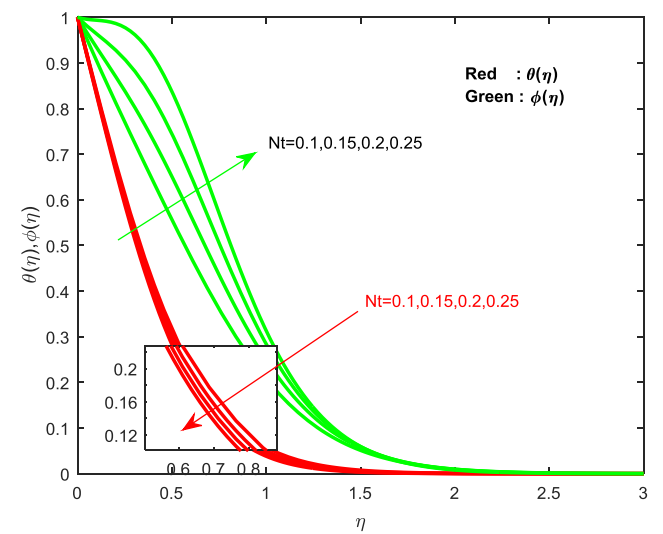

Figure 12. Temperature Profiles $\theta(\eta)$ and Concentration Profiles $\phi(\eta)$ for Different Values of Thermophoresis Parameter $N t$

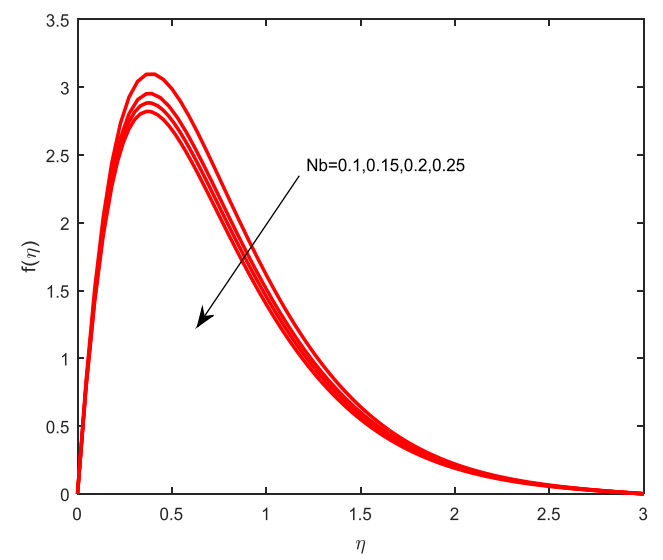

Figure 13. Velocity Profiles $f(\eta)$ for Various Values of Brownian Motion Parameter $\mathrm{Nb}$ 


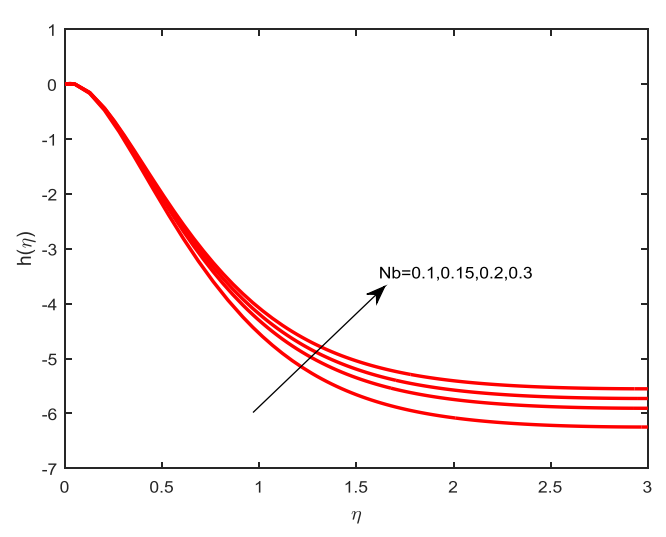

Figure 14. Velocity Profiles $h(\eta)$ for Various Values of Brownian Motion Parameter $\mathrm{Nb}$

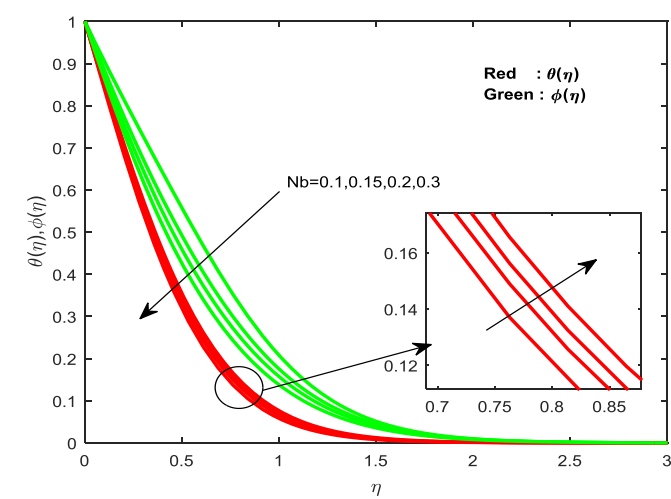

Figure 15. Temperature Profiles $\theta(\eta)$ and Concentration Profiles $\phi(\eta)$ for Different Values of Brownian Motion Parameter $\mathrm{Nb}$

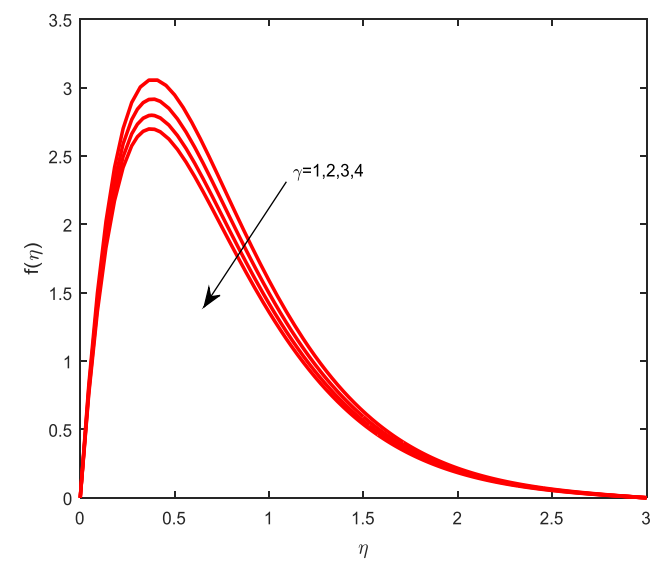

Figure 16. Velocity Profiles $f(\eta)$ for Various Values of Chemical Reaction Parameter $\gamma$

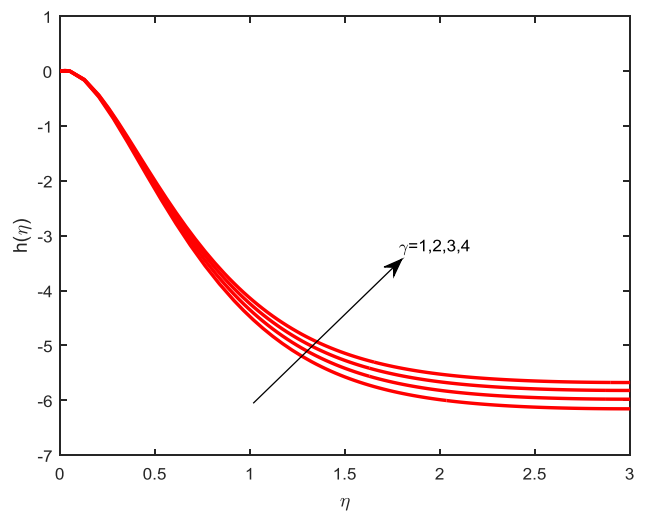

Figure 17. Velocity Profiles $h(\eta)$ for Different Values of Chemical Reaction Parameter $\gamma$

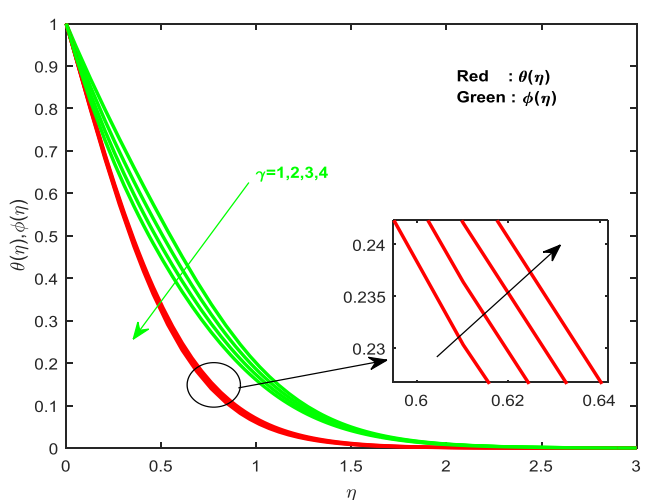

Figure 18. Temperature Profiles $\theta(\eta)$ and Concentration Profiles $\phi(\eta)$ for Different Values of Chemical Reaction Parameter $\gamma$

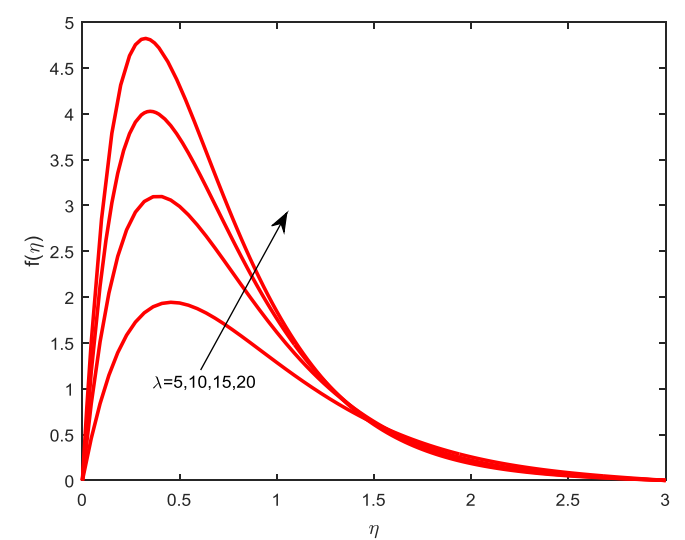

Figure 19. Velocity Profiles $f(\eta)$ for Various Values of Buoyancy Parameter $\lambda$ 


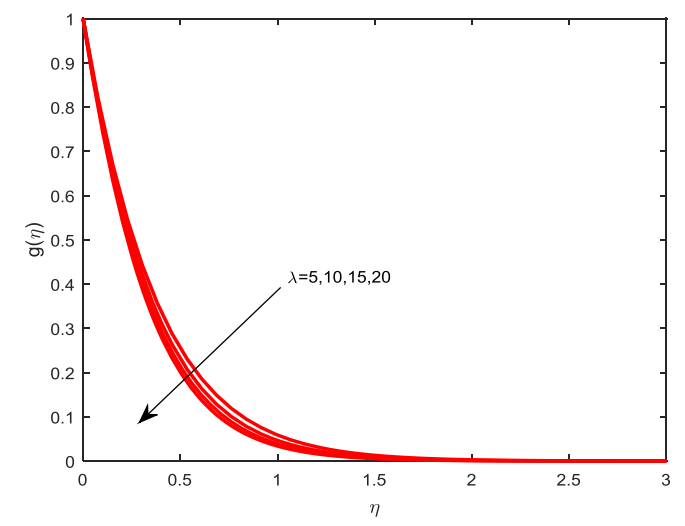

Figure 20. Velocity Profiles $g(\eta)$ for Different Values of Buoyancy Parameter $\lambda$

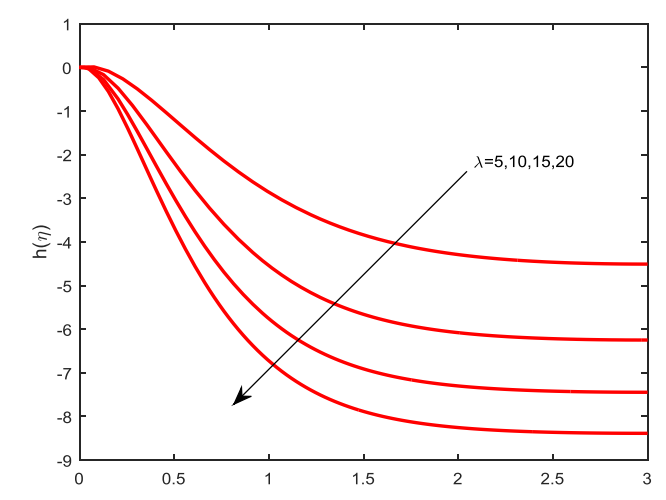

Figure 21. Velocity Profiles $h(\eta)$ for Different Values of Buoyancy

Parameter $\lambda$

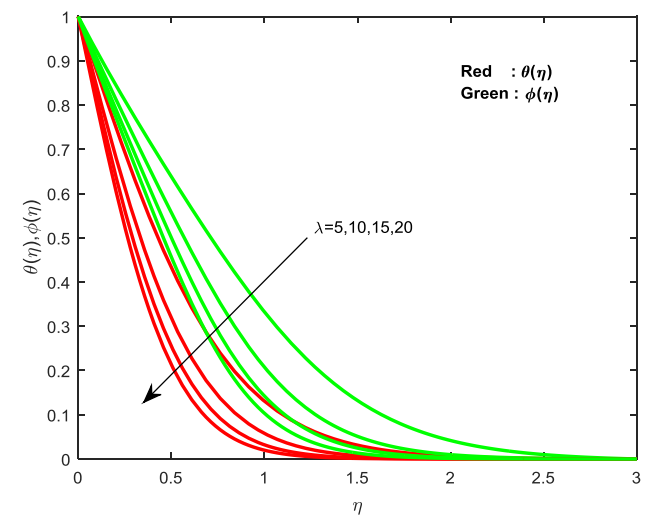

Figure 22. Temperature Profiles $\theta(\eta)$ and Concentration Profiles $\phi(\eta)$ for Different Values of Buoyancy Parameter $\lambda$

Table 10. Comparison of the Present Results for Skin Friction Coefficient and Local Nusselt Number for $\lambda$ when $\operatorname{Pr}=0.7, D a^{-1}=N=N b=N t=0$.

\begin{tabular}{|c|c|c|c|c|c|c|}
\hline$\lambda$ & $\begin{array}{c}\text { Hering } \\
\text { and } \\
\begin{array}{c}\text { Grosh } \\
{[3]} \\
-g^{\prime}(0)\end{array}\end{array}$ & $\begin{array}{c}\text { Mallikarjuna } \\
\text { et al. [32] } \\
-g^{\prime}(0)\end{array}$ & $\begin{array}{c}\text { Present } \\
\text { Results } \\
-g^{\prime}(0)\end{array}$ & $\begin{array}{c}\text { Hering } \\
\text { and } \\
\text { Grosh } \\
{[3]} \\
-\theta^{\prime}(0)\end{array}$ & $\begin{array}{c}\text { Mallikarjuna } \\
\text { et al. [32] } \\
-\theta^{\prime}(0)\end{array}$ & $\begin{array}{c}\text { Present } \\
\text { Results } \\
-\theta^{\prime}(0)\end{array}$ \\
\hline 0 & 0.61592 & 0.61583 & 0.615825 & 0.42852 & 0.42842 & 0.428423 \\
\hline 0.1 & 0.65489 & 0.65492 & 0.654933 & 0.46156 & 0.46141 & 0.461413 \\
\hline 1.0 & 085076 & 085080 & 0850802 & 0.61202 & 0.61213 & 0.612124 \\
\hline 10 & 1.40370 & 1.40363 & 1.403631 & 1.01730 & 1.01748 & 1.017482 \\
\hline
\end{tabular}


Table 2. Variation in Friction Factors, Local Nusselt and Sherwood Numbers

\begin{tabular}{|c|c|c|c|c|c|c|c|c|c|}
\hline M & $\delta$ & $N t$ & $N b$ & $\gamma$ & $\lambda$ & $-h "(0)$ & $-g^{\prime}(0)$ & $-\theta^{\prime}(0)$ & $-\phi^{\prime}(0)$ \\
\hline 1 & & & & & & 43.051760 & 2.673730 & 1.590582 & 0.975622 \\
\hline 2 & & & & & & 39.003511 & 3.292434 & 1.470493 & 0.896427 \\
\hline 3 & & & & & & 36.226669 & 3.826581 & 1.376940 & 0.838854 \\
\hline 4 & & & & & & 34.184172 & 4.301802 & 1.300375 & 0.795086 \\
\hline & 2 & & & & & 28.016437 & 2.158899 & 1.345453 & 0.869418 \\
\hline & 3 & & & & & 36.068126 & 2.420441 & 1.488771 & 0.932337 \\
\hline & 4 & & & & & 43.051760 & 2.673730 & 1.590582 & 0.975622 \\
\hline & 5 & & & & & 49.345191 & 2.915023 & 1.669789 & 1.008123 \\
\hline & & 0.10 & & & & 43.051760 & 2.673730 & 1.590582 & 0.975622 \\
\hline & & 0.15 & & & & 44.769104 & 2.688730 & 1.621340 & 0.693259 \\
\hline & & 0.20 & & & & 46.495936 & 2.703377 & 1.647892 & 0.411026 \\
\hline & & 0.25 & & & & 48.226345 & 2.717664 & 1.670883 & 0.130558 \\
\hline & & & 0.10 & & & 43.051760 & 2.673730 & 1.590582 & 0.975622 \\
\hline & & & 0.15 & & & 41.974409 & 2.664047 & 1.533740 & 1.183496 \\
\hline & & & 0.20 & & & 41.474403 & 2.659429 & 1.494253 & 1.285174 \\
\hline & & & 0.30 & & & 41.040517 & 2.655287 & 1.433917 & 1.386255 \\
\hline & & & & 1 & & 42.704180 & 2.670805 & 1.579360 & 1.072348 \\
\hline & & & & 2 & & 42.056477 & 2.665342 & 1.558209 & 1.256714 \\
\hline & & & & 3 & & 41.466399 & 2.660352 & 1.538678 & 1.430018 \\
\hline & & & & 4 & & 40.927624 & 2.655790 & 1.520640 & 1.593421 \\
\hline & & & & & 5 & 24.499406 & 2.566936 & 1.231501 & 0.812841 \\
\hline & & & & & 10 & 43.051760 & 2.673730 & 1.590582 & 0.975622 \\
\hline & & & & & 15 & 60.212785 & 2.758579 & 1.828685 & 1.090794 \\
\hline & & & & & 20 & 76.495402 & 2.830644 & 2.011638 & 1.181350 \\
\hline
\end{tabular}

\section{Conclusions}

Present study is a numerical investigation of the flow, heat and mass transfer behaviour of magnetohydrodynamic flow towards a vertical rotating cone through porous medium in the presence of thermophoretic and Brownian motion effects. The conclusions of the present study are as follows:

- Magneticfield parameter have tendency to control the flow and reduce the heat and mass transfer rates.

- Thermophoresis and Brownian motion parameter helps to control the heat and mass transfer rates.

- Porosity parameter has capability to enhance the heat and mass transfer rate.

- An increase in chemical reaction parameter reduces the friction factors, heat transfer rate and enhances the mass transfer rate.

- Thermal and concentration boundary layers are highly influenced by magneticfield parameter.

\section{References}

[1] P. Cheng and W. J. Minkowycz, "Free convection about a vertical flat plate embedded in a porous medium with application to heat transfer from a dike", Journal of Geophys. Res., vol. 82, (1977), pp. 2040-2044. 
[2] K. R. Khair and A. Bejan, "Mass transfer to natural convection boundary layer flow driven by heat transfer", Journal of Heat Transfer, vol. 107, (1985), pp. 979-981.

[3] R. G. Hering and R. J. Grosh, "Laminar combined convection from a rotating cone", ASME. Journal of Heat Transfer, vol. 85, (1963), pp. 29-34.

[4] K. Himasekhar, P. K. Sarma and K. Janardhan, "Laminar mixed convection from a vertical rotating cone", International Commu. Heat Mass Transfer, vol. 16, (1989), pp. 99-106.

[5] S. Nadeem and S. Saleem, "Analytical treatmet of unsteady mixed convection MHD flow on a Rotating cone in a rotating frame", Journal of the T. Inst. Of Chem. Eng., vol. 44, (2013), pp. 596-604.

[6] R. Ravindran, S. Roy and E. Momoniat, "Effects of injection on a steady mixed convection boundary layer flow over a vertical cone", International Journal of Numer. Method H., vol. 19, (2009), pp. 432444.

[7] S. Roy, P. Datta and N. C. Mahanti, "Non-similar solution of an unsteady mixed convection flow over a vertical cone with suction or injection", International Journal of Heat Mass Tran., vol. 50, (2007), pp. 181-187.

[8] P. Mohan Krishna, N. Sandeep and V. Sugunamma, "Effects of radiation and chemical reaction on MHD convective flow over a permeable stretching surface with suction and heat generation", Walaliak Journal of Science and Technology, vol. 12, no. 9, (2015), pp. 831-847.

[9] N. Sandeep and C. Sulochana, "Dual solutions for unsteady mixed convection flow of MHD Micropolar fluid over a stretching/shrinking sheet with non-uniform heat source/sink", Engineering Science and Technology, an International Journal, vol. 18, (2015), pp. 738-745.

[10] S. Roy and D. Anilkumar, "Unsteady mixed convection from a rotating cone in a rotating fluid due to the combined effects of thermal and mass diffusion", International Journal of Heat Mass Tran, vol. 47, (2004), pp. 1673-1684.

[11] R. S. R. Gorla, A. Chamkha and K. Ghodeswar, "Natural convective boundary layer flow over a vertical cone embedded in a porous medium saturated with a nanofluid", Journal of Nanofluids, vol. 3, (2014), pp. 65-71.

[12] S. Nadeem and S. Saleem, "Theoretical investigation of MHD nanofluid flow over a rotating cone: An optimal solution”, Inf. Sci. Lett., vol. 3, no. 2, (2014), pp. 55-62.

[13] A. Kaya, "Effects of radiation-conduction interaction on mixed convection from a vertical cone embedded in a porous media with high porosity", Turkish Journal of Eng. Env. Sci., vol. 38, (2014), pp. 51-63.

[14] B. R. Sharma, "Debozani Borgohain, Soret and Dufour effects on chemically reacting MHD mixed convection flow from a rotating vertical cone in a porous medium", International Journal of Sci. Res. Dev., vol. 3, no. 4, (2015), pp. 1713-1720.

[15] S. M. M. El. Kabeir, M. A. El-Hakiem and A. M. Rashad, "Group method analysis for the effect of radiation on MHD coupled heat and mass transfer natural convection flow water vapor over a vertical cone through a porous medium”, International Journal of Appl. Math. Mech., vol. 3, (2007), pp. 35-53.

[16] E. M. A. Elbashbeshy, D. M. Yassmin and A. A. Dalia, "Heat transfer over an unsteady porous stretching surface embedded in a porous medium with variable heat flux in the presence of heat source or sink", Afr. Journal of Math. Comp. Sci. Res., vol. 3, no. 5, (2010), pp. 68-73.

[17] W. T. Cheng and C. H. Lin, "Melting effect on mixed convective heat transfer with aiding and opposing external flows from the vertical plate in a liquid saturated porous medium", International Journal of Heat Mass Transfer, vol. 50, (2007), pp. 3026-3034.

[18] O. D. Makinde and A. Ogulu, "The effect of thermal radiation on the heat and mass transfer flow of a variable viscosity fluid past a vertical porous plate permeated by a transverse magnetic field", Chem. Eng. Commu., vol. 195, no. 12, (2008), pp. 1575-1584.

[19] M. M. Rashidi, S. Abelman and N. Freidooni Mehr, "Entropy generation in steady MHD flow due to a rotating porous disk in a nanofluid", International Journal of Heat Mass Transfer, vol. 62, (2013), pp. $15-525$.

[20] B. R. Rout, S. K. Parida and S. Panda, "MHD heat and mass transfer of chemical reaction fluid flow over a moving vertical plate in presence of heat source with convective surface boundary condition", Int. J. chem. Eng., (2013), Article ID 296834. http://dx.doi.org/10.1155/2013/296834.

[21] C. S. K. Raju, N. Sandeep, C. Sulochana, V. Sugunamma and M. Jayachandra Babu, "Radiation, Inclined Magnetic field and Cross-Diffusion effects on flow over a stretching surface", Journal of the Nigerian Mathematical Society, vol. 34, (2015), pp. 169-180.

[22] S. K. Parida, M. Acharya, G. C. Dash and S. Panda, "MHD heat and mass transfer in a rotating system with periodic suction", Arab. Journal of sci. Eng., vol. 36, no. 6, (2011), pp. 1139-1151.

[23] M. Kumari, I. Pop and G. Nath, "Mixed convection along a vertical cone", Int. Comm. Heat Mass Transfer, vol. 16, (1989), pp. 247-255.

[24] H. S. Takhar, A. J. Chamkha and G. Nath, "Unsteady mixed convection flow from a rotating vertical cone with magnetic field", Heat Mass Transfer, vol. 39, (2003), pp. 297-304.

[25] M. E. Ali, "The effect of variable viscosity on mixed convection heat transfer along a vertical moving surface", International Journal of Therm. Sci., vol. 45, (2006), pp. 60-69. 
[26] A. M. Rashad, M. A. El-Hakiem and M. M. M. Abdou, "Natural convection boundary layer flow of a non-Newtonian fluid about a permeable vertical cone embedded in a porous medium saturated with a nanofluid", Computers and Mathematics with Applications, vol. 62, (2011), pp. 3140-3151.

[27] B. Rushi Kumar and R. Sivaraj, "MHD viscoelastic fluid non-Darcy flow over a vertical cone and a flat plate", International Commu. Heat Mass Transfer, vol. 40, (2013), pp. 1- 6.

[28] V. Ramachandra Prasad, B. Vasu and O. Anwar Beg, "Thermo-diffusion and diffusion-thermo effects on MHD free convection flow past a vertical porous plate embedded in a non-Darcian porous medium", Chemical engineering Journal, vol. 173, (2011), pp. 598-606.

[29] F. S. Ibrahim, A. M. Elaiw and A. A. Bakr, "Effect of the chemical reaction and radiation absorption on the unsteady MHD free convection flow past a semi infinite vertical permeable moving plate with heat source and suction", Commu. Nonlinear Sci. Num. Simulation, vol. 13, no. 6, (2008), pp. 1056-1066.

[30] A. Mahdy, "Effect of chemical reaction and heat generation or absorption on double-diffusive convection from a vertical truncated cone in porous media with variable viscosity", Int. Commu. In Heat and Mass Transfer, vol. 37, no. 5, (2010), pp. 548-554.

[31] U. N. Das, R. Deka and V. M. Soundalgekar, "Effects of mass transfer on flow past an impulsively started infinite vertical plate with constant heat flux and chemical reaction", Forschungim Ingenieurwesen/ Engineering Research, vol. 60, no. 10, (1994), pp. 284-287.

[32] B. Mallikarjuna, A. M. Rashad, A. J. Chamkha and S. Hariprasad Raju, "Chemical reaction effects on MHD convective heat and mass transfer flow past a rotating vertical cone embedded in a variable porosity regime", Afrika Mathematika, DOI 10.1007/s13370-015-0372-1.

\section{Authors}

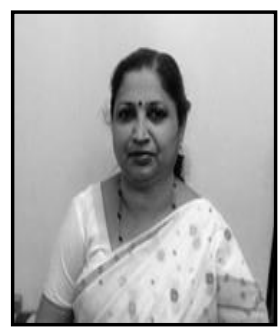

Prof. C. Sulochana obtained her M.Sc., and M.Phil., degrees from Gulbarga University, Gulbarga, Karnataka and Ph.D degree from Sri Krishna Devaraya University, Anantapur. She produced 6 Ph.D and 7 M.Phil, degrees. She published more than 35 papers in reputed scientific journals and presented papers in national and international conferences. She handled various prestigious positions at Gulbarga University. At present she is serving as a professor at Department of Mathematics, Gulbarga University, Gulbarga. Her area of interest is Fluid Mechanics.

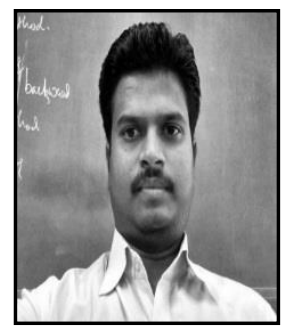

Mr. G.P. Ashwinkumar obtained his M.Sc. degree from Central University of Karnataka, Gulbarga. Presently he is pursuing Ph.D degree under the guidance of Prof. C. Sulochana in Department of Mathematics, Gulbarga University, Gulbarga, Karnataka. His area of interest is Fluid Mechanics.

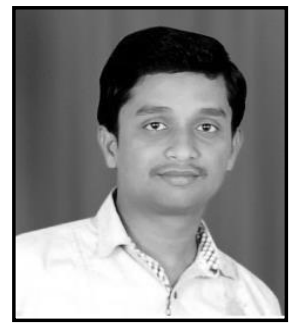

Dr. N. Sandeep obtained his M.Sc., and Ph.D. degrees from Sri Venkateswara University, Tirupati, M.Phil degree from Madurai Kamaraj University, Madurai. He published more than 30 papers in reputed scientific journals and presented papers in national and international conferences. At present he is serving as a Post Doctoral Fellow under UGC Dr.D.S.Kothari Fellowship Scheme at Department of Mathematics, Gulbarga University, Gulbarga. His area of interest is Fluid Mechanics. 
International Journal of Advanced Science and Technology Vol.86 (2016) 